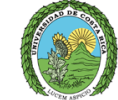

UNIVERSIDAD DE

COSTARICA
Artículo científico

Volumen 30(2):367-380. Mayo-agosto, 2019

e-ISSN 2215-3608, doi:10.15517/am.v30i2.31760

https://revistas.ucr.ac.cr/index.php/agromeso/index

\title{
Bacterias endófitas de Capsicum frutescens antagónicas a Fusarium spp. ${ }^{1}$
}

\section{Endophytic bacteria of Capsicum frutescens antagonistic to Fusarium spp.}

\author{
Martha Lucia Velasco-Belalcázar², Carlos Alberto Hernández-Medina², Eyder Daniel Gómez-López², \\ Celina Torres-Gonzales ${ }^{3}$, Paola Andrea Caro-Hernández ${ }^{4}$
}

1 Recepción: 26 de abril, 2018. Aceptación: 17 de octubre, 2018. Este trabajo formó parte de la tesis de Maestría de la primera autora, la cual fue financiada por el macroproyecto de regalías "Desarrollo de un sistema agroindustrial rural competitivo en una bioregión del Valle del Cauca" de la Universidad del Valle, Cali, Colombia, y contó con la financiación de la Universidad Nacional de Colombia sede Palmira y la Universidad Libre, Seccional Cali, Colombia.

2 Universidad Nacional de Colombia, Facultad de Ciencias Agropecuarias. Grupo de investigación en Protección Vegetal para el Mejoramiento de la Productividad. A. A. 237. Palmira, Valle del Cauca, Colombia. malvelascobe@unal.edu.co; caahernandezme@unal.edu.co (https:// orcid.org/0000-0002-4002-4109); eydgomezlo@unal.edu.co

3 Universidad del Valle, Facultad de Ciencias, A.A. 25360 Cali, Valle del Cauca, Colombia. celina.torres@correounivalle.edu.co

4 Universidad Libre, Seccional Cali, Grupo Microambiente Libre, A.A. 1040 Cali, Valle del Cauca, Colombia. paolaa.caro@unilibre.edu.co (https://orcid.org/0000-0003-4362-0405).

\section{Resumen}

Introducción. En las últimas décadas se ha incrementado el interés por el cultivo de ají tabasco en Colombia, sin embargo, se han observado limitantes en la producción, debido a problemas fitosanitarios. Especies del género Fusarium ocasionan pudriciones de raíz y tallo con pérdidas cuantiosas para los agricultores, por lo cual es importante encontrar alternativas de manejo a la marchitez vascular ocasionada por Fusarium spp. Objetivo. El objetivo de este trabajo fue aislar y caracterizar bacterias endófitas, con potencial antagónico in vitro frente a Fusarium spp., en plantas de ají tabasco (Capsicum frutescens var. Tabasco). Materiales y métodos. En el periodo entre febrero de 2014 a febrero de 2016, se evaluó la capacidad antagónica in vitro de 68 aislados bacterianos endófitos de tejido foliar C. frutescens, provenientes de dos municipios del Valle del Cauca, Colombia. Las bacterias aisladas fueron enfrentadas a seis aislamientos patogénicos de Fusarium spp, mediante el método de crecimiento dual. Resultados. Cincuenta de los aislados bacterianos presentaron porcentajes de inhibición frente al menos un aislado de Fusarium y de estos, dieciséis presentaron porcentajes de inhibición por encima del $40 \%$. La morfología, perfil bioquímico y caracterización molecular, permitieron determinar que los aislados identificados como Bacillus subtillis y Pseudomonas aeruginosa, mostraron promedios de inhibición entre 62 y $89 \%$, altos con respecto a los reportados anteriormente en otros estudios. Además, se destacan como nuevos hallazgos de bacterias asociadas al tejido vegetal endófito de C.frutescens, en el Valle del Cauca, Colombia, a las especies Enterobacter cloacae, Microbacterium arborescens, y Stenotrophomonas maltophilia. Conclusión. Estos resultados constituyen una fuente potencial para el manejo de patógenos y mejoramiento de la productividad en el ají tabasco en Colombia.

Palabras clave: ají, antagonismo, control biológico, fitopatógeno.

\begin{abstract}
Introduction. In the last decades, there has been an increased on the interest of Tabasco Chilli pepper cultivation in Colombia; however, production limitation has been observed due to phytosanitary problems. Species of the
\end{abstract}


genus Fusarium cause root and stem rots with large losses for farmers; whereby, it is important to find alternatives to vascular wilt managment caused by Fusarium spp. Objective. The objective of this research was to isolate and characterize endophytic foliar bacteria with in vitro antagonistic potential against Fusarium spp. in Tabasco Chili pepper (Capsicum frutescens) plants. Materials and methods. In the period between February 2014 and February 2016 , the in vitro antagonistic capacity of 68 bacterial endophytes of leaf tissue $C$. frutescens, from two municipalities of Valle del Cauca, Colombia, was evaluated. The isolated bacteria were confronted with six pathogenic isolates of Fusarium spp. by dual growth method. Results. Fifty of the bacterial isolates showed percentages of inhibition against at least one Fusarium isolate, and of these sixteen had percentages of inhibition above $40 \%$. The morphology, biochemical profile and molecular characterization allowed to determine that the isolates identified as Bacillus subtillis and Pseudomonas aeruginosa showed inhibition averages between 62 and 89\%, high averages compared to those previously reported in other studies. In addition, stand out as new findings of bacteria associated with endophytic plant tissue of $C$. frutescens, in Valle del Cauca, Colombia, the Enterobacter cloacae, Microbacterium arborescens, and Stenotrophomonas maltophilia species. Conclusion. These results constitute a potential source for pathogen management and productivity improvement in tabasco chili pepper in Colombia.

Keywords: Pepper, antagonism, biological control, plant pathogens.

\section{Introducción}

Se estima que los cultivos de ají en Colombia aumentan a una tasa del $13 \%$ anual, lo que indica grandes posibilidades en el incremento de su producción, lo que significa una alternativa para la diversificación y generación de ingresos para pequeños agricultores (Ochoa y Lundy, 2002). El cultivo de ají es afectado por numerosas enfermedades de etiología microbiana (Guigón-López y González-González, 2001; Andrés-Ares et al., 2005; Islek y Turkilmaz-Unal, 2015), entre las que se encuentra la podredumbre de raíz y tallo, causada por Fusarium oxysporum (Perez-Hernández et al., 2014). El hongo penetra en la planta obstruyendo los vasos del xilema que colapsan e impiden el flujo de nutrientes y agua que deberían llegar a las hojas, estas comienzan a marchitarse y el proceso puede continuar hasta que la planta completa se marchita y muere (Agrios, 2005). Fusarium, además, produce un grupo de enzimas que degradan la pared celular de la planta, facilitándole el proceso de infección y afectando otros órganos como tallos, flores y frutos (Turlier et al., 1994; González et al., 2012).

Una alternativa amigable y de bajo costo para el control de hongos fitopatógenos es el empleo de bacterias endófitas. Estas bacterias se encuentran dentro de los tejidos de raíces, tallos y hojas, de casi todas las plantas en el planeta (Smith et al., 2008), ejerciendo un importante rol en el metabolismo y fisiología de la planta, y por tanto, estableciendo una estrecha relación con el hospedador sin causar perjuicios (Reinhold-Huker y Hurek, 1998; Kloepper et al., 1999; Pérez et al., 2013). Algunas bacterias endófitas hacen parte de las bacterias promotoras de crecimiento vegetal (PGPB, por sus siglas en inglés de plant promoting bacteria), y al igual que las bacterias rizosféricas PGPB, presentan mecanismos directos e indirectos, o una mezcla de estos, que benefician a las plantas en su desarrollo (Glick, 2012). Los mecanismos directos de promoción de crecimiento vegetal, incrementan la disponibilidad de nutrientes en la rizósfera, al influir en el metabolismo de las plantas y mejorar su nutrición. En bacterias endófitas se puede mencionar, la fijación de nitrógeno (Puri et al., 2018), la solubilización de fósforo (Walia et al., 2017), la producción de sideróforos para la adquisición de hierro (Rajkumara et al., 2009) o la regulación y síntesis de hormonas estimuladoras de crecimiento vegetal (Santoyo et al., 2015). Los mecanismos indirectos se caracterizan por ocasionar la inhibición o eliminación de los fitopatógenos o prevenir el daño que provocan en las plantas, ya sea mediante la producción de antibióticos (Glick, 2015; Santoyoa et al., 2015) u otros metabolitos (Brader et al., 2014), síntesis de compuestos volátiles inhibidores de patógenos, competencia 
de nutrientes o del espacio en el nicho ecológico, y estimulación de las defensas naturales de la planta, mediante mecanismos conocidos como resistencia sistémica inducida (Santoyo et al., 2015).

En plantas de ají se han aislado una gran cantidad de bacterias endófitas (Narayan et al., 2013; Amaresan et al., 2014), dentro de las cuales, cepas de Bacillus tequilensis, Burkholderia cepacia y Peudomonas aeruginosa, han demostrado capacidad antagónica frente a F. oxysporum y otros hongos fitopatógenos (Narayan et al., 2013). El género Bacillus se ha reportado como un antagonista dominante frente a Fusarium tipo silvestre (Edward et al., 2013; Amaresan et al., 2014; Muhammad et al., 2016), al igual que diferentes especies de Pseudomonas (Barquero et al., 2016; Muhammad et al., 2016).

Aunque las rizobacterias y bacterias endófitas presentan los mismos mecanismos para la promoción de crecimiento vegetal, las bacterias endófitas están menos afectadas por el estrés ambiental, por lo que podrían presentar mayor ventaja ecológica al emplearse como biocontroladores (Sturz y Nowak, 2000; Peña y Reyes, 2007; Santoyo et al., 2015). Teniendo en cuenta lo anterior, el aislamiento, identificación y caracterización de bacterias endófitas con capacidad antagónica frente a hongos fitopatógenos es un campo amplio por explorar, que tendría un gran potencial en la agricultura para el desarrollo sostenible. El objetivo de este estudio fue aislar y caracterizar bacterias endófitas foliares, con potencial antagónico in vitro frente a Fusarium spp., en plantas de ají tabasco (Capsicum frutescens var. Tabasco).

\section{Materiales y métodos}

El estudio se llevó a cabo en el periodo comprendido entre febrero de 2014 a febrero de 2016.

\section{Colecta de muestras vegetales}

Las muestras se colectaron en el tercer y octavo mes del cultivo, en dos fincas productoras de ají tabasco (Capsicum frutescens Var. Tabasco), de los municipios de Guacarí (345’21,68”N - 76¹1'57,72”O; 971 msnm) y Bolívar (4²5’00,47’N - 7608'54,53”O; 939 msnm), Valle del Cauca, Colombia. Se realizó un muestreo total aleatorio donde se obtuvo, por localidad, tejido foliar de quince plantas establecidas como sanas, siguiendo el protocolo descrito por Morales-Rondón y Rodríguez-González (2006), con modificaciones. Se tomaron tres hojas sanas por cada punto cardinal por planta, y estas se depositaron en bolsas de papel, debidamente rotuladas, para llevarlas al Laboratorio de Investigaciones Microbiológicas de la Universidad del Valle y al Laboratorio de Microbiología Ambiental, de la Universidad Libre, Seccional Cali, para su procesamiento.

\section{Aislamiento de bacterias endófitas}

De las muestras colectadas de cada planta se seleccionaron seis hojas sanas y se esterilizaron siguiendo el protocolo de Nongkhlaw et al. (2014), con algunas modificaciones. Se lavaron con hipoclorito de sodio al $1 \%$ (1 min), alcohol al $70 \%$ (1 min), seguido de un enjuague con agua destilada estéril y un secado sobre toallas de papel estéril (30 min). Posteriormente, las seis hojas se maceraron, el macerado fue diluido en $10 \mathrm{ml}$ de agua destilada estéril; de esta suspensión se tomaron $100 \mu \mathrm{l}$ que se sembraron en superficie sobre cajas Petri con agar nutritivo. Los cultivos se incubaron a $28^{\circ} \mathrm{C}$ durante $24-48 \mathrm{~h}$. Las colonias obtenidas se purificaron, codificaron y mantuvieron a $-81{ }^{\circ} \mathrm{C}$ para su posterior caracterización e identificación.

Cada aislamiento bacteriano, se rotuló de acuerdo con el siguiente código: E (endófito foliar), $\mathrm{P}$ ( $\mathrm{n}^{\circ}$ planta del que fue colectado), B1 (Bolívar primer muestreo, tercer mes del cultivo), B2 (Bolívar segundo muestreo, octavo mes del cultivo), G1 (Guacarí primer muestreo, tercer mes del cultivo), o G2 (Guacarí segundo muestreo, 
octavo mes del cultivo), de acuerdo al lugar y mes de colecta y $\mathrm{M}$ (morfotipo o número de colonia bacteriana con características morfológicas distintivas y diferentes de las demás colonias presentes en la caja Petri analizada).

\section{Actividad antagónica de los aislados}

El potencial antagónico in vitro se llevó a cabo en el Laboratorio de Microbiología Ambiental de la Universidad Libre, Seccional Cali. Para ello, se llevó a cabo la técnica de cultivo dual, en la que cada una de las bacterias endófitas se enfrentó de forma directa a seis aislamientos patogénicos, identificados mediante caracteres morfométricos descritos por Leslie y Summerell (2006) como Fusarium spp., obtenidos e identificados molecularmente en otros estudios realizados en el Laboratorio de Investigaciones Microbiológicas de la Universidad del Valle y el Laboratorio de Fitopatología de la Universidad Nacional de Colombia sede Palmira (Clavijo-Castro, 2014; VelascoBelalcazar, 2016). Como controles se emplearon los aislados de Fusarium spp. en cultivo puro. Los aislados patogénicos de Fusarium empleados fueron: FS-09 proveniente de Darien, FS-70 proveniente de Ceunp, FS-58 proveniente de Yotoco, FS-52 y FS-54 provenientes de Yumbo, obtenidas por Clavijo-Castro (2014). Además, se incluyó el aislado FB proveniente de Bolívar, obtenido por Velasco-Belalcazar (2016). La patogenicidad de los aislados se corroboró (FS-70, FS-58, FS-54, FS-52 y FS-09) y determinó (FB) mediante el método de inoculación por inmersión de raíces y aplicación del inóculo a una concentración de $10^{6}$ esporas.ml ${ }^{-1}$, siguiendo el protocolo utilizado por Clavijo-Castro (2014). Una vez confirmada la patogenicidad de los mismos, se procedió a realizar el enfrentamiento con las bacterias endófitas. Los ensayos se realizaron por triplicado, sembrando en agar papa dextrosa (PDA), e incubando a $28^{\circ} \mathrm{C}$ de 7 a 28 días.

El porcentaje de inhibición del crecimiento del patógeno se calculó empleando la fórmula descrita por Acosta et al. (2007).

$\%$ inhibición $=((R 1-R 2) / R 1) * 100$.

Donde R2 representó el crecimiento del hongo en presencia de bacterias y R1 el crecimiento del control.

El comportamiento de inhibición mostrado por los diferentes aislamientos se agrupó de la siguiente manera (Benítez et al., 2007):

- Negativo: ausencia de zona de inhibición o un porcentaje menor de $10 \%$ y crecimiento normal del patógeno, de forma similar al control.

- Baja: ausencia de zona de inhibición o con un porcentaje entre 10 - $39 \%$ y con disminución en el crecimiento del patógeno.

- Media: ausencia de zona de inhibición o un porcentaje entre 40 - 69 \% y con disminución en el crecimiento del patógeno.

- Alta (positiva): presencia de zona de inhibición definida o en un porcentaje entre 70 - $100 \%$.

Finalmente, las bacterias que presentaron porcentajes de inhibición superior al $10 \%$-frente a por lo menos dos aislados del patógeno, se sometieron a una caracterización bioquímica y molecular.

\section{Caracterización bioquímica}

Las bacterias endófitas con potencial antagónico se caracterizaron e identificaron mediante las pruebas bioquímicas API20E para bacterias Gram negativas y API50CHB para bacterias Gram positivas (BioMerieux®), con base en los protocolos de la casa comercial. La identificación se realizó mediante la comparación de los resultados obtenidos con el banco de datos de BioMerieux ${ }^{\circledR}$, según el sistema de códigos en software proporcionado por la casa comercial. 
Las bacterias caracterizadas como Gram negativas, además se sembraron en el medio King B y agar Cetrimide para evaluar la habilidad de producir sideróforos.

\section{Identificación molecular}

La identificación molecular se llevó a cabo en el Laboratorio de Diagnóstico Vegetal de la Universidad Nacional de Colombia, sede Palmira. Para ello, se realizó el análisis de la secuencia del gen 16S rRNA, y se utilizó para su amplificación por PCR los primers universales descritos por Weisburg et al. (1991):

fD1(5'-CCGAATTCGTCGACAACAGAGTTTGATCCTGGCTCAG-3') rD1(5'CCCGGGATCCAAGCTTA AGGAGGTGATCCAGCC-3').

El ADN total fue extraído de acuerdo con el método de CTAB propuesto por Merino y Giusiano (2011). La amplificación se realizó siguiendo los ciclos de temperatura que se describen a continuación: desnaturalización inicial a $95{ }^{\circ} \mathrm{C}$ por $2 \mathrm{~min}$, seguida de 30 ciclos a $95^{\circ} \mathrm{C}$ por $30 \mathrm{~s}, 55^{\circ} \mathrm{C}$ por $4 \mathrm{~min}, 72{ }^{\circ} \mathrm{C}$ por 4 min, y una extensión final del ADN a $72{ }^{\circ} \mathrm{C}$ durante $5 \mathrm{~min}$. Los productos de PCR se purificaron y secuenciaron por Macrogen Korea. Las secuencias obtenidas se compararon con secuencias similares en el GenBank utilizando BLAST. Para el análisis de las secuencias y construcción del árbol filogenético se utilizaron los programas Bioedit 7.0 y MEGA 6.

\section{Resultados}

\section{Potencial antagónico de bacterias endófitas frente a Fusarium spp.}

En las sesenta muestras de tejido foliar de ají tabasco procesadas, se obtuvieron un total de 68 aislados de bacterias endófitas, de los cuales cincuenta mostraron potencial antagónico, en nivel bajo, medio o alto de inhibición, frente a por lo menos uno de los aislados de Fusarium spp. El mayor número de aislados de bacterias endófitas con potencial antagónico se obtuvo en el municipio de Bolívar, con veintinueve aislados, mientras que en Guacarí se obtuvo un total de veintiún aislados.

De los cincuenta aislados endófitos bacterianos obtenidos, dieciséis mostraron un porcentaje de inhibición promedio mayor al $40 \%$ (medio y alto); y de estos los aislados EP5G1M10, EP9G2M18, EP8G1M10, EP3B1M4 sobresalieron con promedios mayores al 70 \% (Cuadro 1). El aislado EP5G1M10 se destacó por encima de los demás, mostrando potenciales de inhibición, frente a todos los Fusarium spp. evaluados, con porcentajes mayores al $76 \%$ en todos los casos.

\section{Caracterización e identificación bioquímica y molecular de bacterias endófitas con potencial de antagonismo.}

Las bacterias endófitas con un porcentaje mayor al $10 \%$ frente a por lo menos dos de los seis aislados de Fusarium spp., se caracterizaron mediante pruebas bioquímicas rápidas e identificaron mediante el análisis de la secuencia del gen 16S rRNA (Cuadro 2).

Los resultados ofrecidos por el sistema API 20E y 50CHB, mostraron que, de los veinticuatro aislados identificados, los géneros más representativos fueron Pseudomonas y Bacillus, con nueve y siete aislados, respectivamente. Además, se encontró que los perfiles bioquímicos indicaron la presencia de los géneros: Stenotrophomonas, Enterobacter, Aneurinibacillus y Geobacillus, este último con un porcentaje bajo de identificación.

Los aislados antagónicos bacterianos identificados como Pseudomonas EP9G2M18, EP9G1M16, EP8G1M10, EP10G1M9 y EP15G1M16 (Cuadro 2), sembrados en los medios selectivos Cetrimide y King B, produjeron sideróforos como piocianinas, pioverdinas y fluoresceínas; evidenciados por los pigmentos verdosos, azules o amarillos que se visualizaron cuando se expusieron a la luz UV. 
Velasco-Belalcázar et al.: Bacterias con capacidad antagónica a Fusarium sp.

Cuadro 1. Promedio del porcentaje de inhibición de bacterias endófitas foliares de Capsicum frutescens, con potencial antagónico frente a los aislados de Fusarium spp. Valle del Cauca, Colombia. 2016.

Table 1. Average percentage of inhibition of endophytic foliar bacteria of Capsicum frutescens, with antagonistic potential against pathogenic Fusarium spp. isolates. Valle del Cauca, Colombia. 2016.

\begin{tabular}{|c|c|c|c|c|c|c|c|c|}
\hline \multirow{2}{*}{$\begin{array}{l}\text { Código del } \\
\text { aislamiento }\end{array}$} & \multirow[t]{2}{*}{ Género bacteriano } & \multirow{2}{*}{$\begin{array}{c}\text { Lugar de } \\
\text { aislamiento }\end{array}$} & \multicolumn{6}{|c|}{ Promedio del porcentaje de inhibición a Fusarium spp. } \\
\hline & & & FS-70 ${ }^{\mathrm{a}}$ & FS-58 ${ }^{\mathrm{a}}$ & FS-54 ${ }^{\text {a }}$ & FS-52 ${ }^{\mathrm{a}}$ & FS-09 & $\mathbf{F B}^{\mathbf{a}}$ \\
\hline EP3G2M4 & Bacillus sp. & Guacari & $67( \pm 0)$ & $33( \pm 0)$ & 0 & 0 & 0 & $63( \pm 4,2)$ \\
\hline EP5G1M9 & Enterobacter sp. & Guacari & $50( \pm 7,9)$ & $13( \pm 3,0)$ & 0 & $17( \pm 7,9)$ & $33( \pm 22,0)$ & $8( \pm 3,4)$ \\
\hline EP5G1M10 $^{\mathrm{b}}$ & Bacillus sp. & Guacari & $76( \pm 3,1)$ & $77( \pm 2,0)$ & $87( \pm 3,1)$ & $82( \pm 6,3)$ & $83( \pm 2,0)$ & $86( \pm 11,0)$ \\
\hline EP6G1M14 & Enterobacter sp. & Guacari & 0 & $64,4( \pm 3,1)$ & 0 & 0 & $28( \pm 8,0)$ & 0 \\
\hline EP10G1M9 & Pseudomonas sp. & Guacari & $50( \pm 1,6)$ & $22( \pm 0)$ & 0 & 0 & 0 & 0 \\
\hline EP8G1M10 & Pseudomonas sp. & Guacari & $71( \pm 3,0)$ & $80( \pm 3,0)$ & $62( \pm 6,0)$ & $69( \pm 3,2)$ & $67( \pm 0)$ & $89( \pm 0)$ \\
\hline EP9G2M18b & Pseudomonas sp. & Guacari & $67( \pm 0)$ & $71( \pm 3,0)$ & $70( \pm 4,7)$ & $68( \pm 1,6)$ & $67( \pm 0)$ & $71( \pm 0)$ \\
\hline EP9G1M16 & Pseudomonas sp. & Guacari & $44( \pm 0)$ & $19( \pm 5,0)$ & $27( \pm 0)$ & $34( \pm 7,9)$ & 0 & $33( \pm 0)$ \\
\hline EP15G1M16 & Pseudomonas sp. & Guacari & $66,7( \pm 0)$ & $11,1( \pm 0,8)$ & $38,9( \pm 7,9)$ & 0 & 0 & $44( \pm 0)$ \\
\hline EP2G2M10 & Pseudomonas sp. & Guacari & $20( \pm 3,1)$ & 0 & 0 & $13( \pm 3,1)$ & $20( \pm 3,0)$ & $22( \pm 6,3)$ \\
\hline EP7G2M15 & Stenotrophomonas sp. & Guacari & 0 & 0 & $37( \pm 5,0)$ & $22,2( \pm 0)$ & 0 & $26( \pm 4,7)$ \\
\hline EP1B2M1 & Bacillus sp. & Bolivar & $47( \pm 3,1)$ & $28( \pm 1,6)$ & 0 & 0 & $27( \pm 3,1)$ & $20( \pm 3,1)$ \\
\hline EP1B2M4 & Bacillus sp. & Bolivar & $42( \pm 3,1)$ & $56( \pm 0)$ & $28( \pm 1,6)$ & $18( \pm 9,4)$ & $42( \pm 6,3)$ & $44( \pm 0)$ \\
\hline EP2B2M4 & Microbacterium sp. & Bolivar & $61( \pm 7,9)$ & $66( \pm 14,1)$ & $48( \pm 20,4)$ & $33( \pm 3,1)$ & $48( \pm 11,0)$ & $46( \pm 2,0)$ \\
\hline $\mathrm{EP} 3 \mathrm{~B} 1 \mathrm{M} 4^{\mathrm{b}}$ & Bacillus sp. & Bolivar & $87( \pm 3,1)$ & $59( \pm 4,7)$ & $72( \pm 1,6)$ & $56( \pm 0)$ & $76( \pm 3,1)$ & $78( \pm 0)$ \\
\hline EP4B2M16 & Bacillus sp. & Bolivar & $44,4( \pm 0)$ & $69( \pm 6,3)$ & $48,9( \pm 3,1)$ & $48( \pm 10,9)$ & $52( \pm 2,0)$ & $83( \pm 7,9)$ \\
\hline EP5B2M21 & Bacillus sp. & Bolivar & 0 & $15,6( \pm 0)$ & $40( \pm 0)$ & $38,9( \pm 7,9)$ & 0 & $24( \pm 3,0)$ \\
\hline ЕР8B2M28 & Stenotrophomonas sp. & Bolivar & $52( \pm 5,0)$ & $58( \pm 3)$ & $51,1( \pm 0)$ & $37( \pm 4,7)$ & $50( \pm 8,0)$ & $89( \pm 0)$ \\
\hline ЕР3В2M13 & Pseudomonas sp. & Bolivar & 0 & 0 & $27,8( \pm 9,0)$ & 0 & $22,2( \pm 0)$ & $24,4( \pm 0)$ \\
\hline EP5B2M17 & Pseudomonas sp. & Bolivar & $25,6( \pm 5,0)$ & 0 & $15,6( \pm 0)$ & $11( \pm 0)$ & 0 & $26( \pm 1,6)$ \\
\hline EP5B2M7 & Pseudomonas sp. & Bolivar & 0 & $24( \pm 3,0)$ & $19( \pm 5,0)$ & $13( \pm 3,1)$ & $33( \pm 0)$ & $33( \pm 0)$ \\
\hline EP1B1M8 & Microbacterium sp. & Bolivar & $8( \pm 4,7)$ & $11( \pm 0,8)$ & $14( \pm 5,0)$ & $6( \pm 2,0)$ & $22,2( \pm 0)$ & 0 \\
\hline EP2B2M7 & Bacillus sp. & Bolivar & 0 & $19( \pm 3,5)$ & 0 & $12( \pm 5,3)$ & $27( \pm 3,0)$ & $18( \pm 0)$ \\
\hline EP4B2M18 & Bacillus sp. & Bolivar & 0 & 0 & $25,6( \pm 1,6)$ & $30( \pm 10,9)$ & $27,8( \pm 7,9)$ & $18( \pm 0)$ \\
\hline
\end{tabular}

a. Aislados patogénicos de Fusarium spp. obtenidos en el Valle del Cauca (Clavijo-Castro, 2014). Colección de referencia de la Universidad Nacional de Colombia sede Palmira FS-70, FS-58, FS-54, FS-52, FS-09 y FB obtenido en el municipio de Bolívar Valle. b. Bacterias endófitas con mayor promedio de porcentaje de inhibición frente a Fusarium spp. / a. Pathogenic isolates of Fusarium spp. obtained in the Valle del Cauca (Clavijo-Castro, 2014). Collection of reference of the National University of Colombia Palmira headquarters FS-70, FS-58, FS-54, FS-52, FS-09 and FB obtained in the municipality of Bolívar Valle. b. Endophytic bacteria with the highest average percentage of inhibition against Fusarium spp.

El análisis de las secuencias del gen 16S rRNA (Figura 1), mostró a los géneros Pseudomonas y Bacillus como los más frecuentes y biodiversos en especies. Nueve de los aislados correspondieron al género Pseudomonas, dentro de los cuales se encontraron las especies P. aeruginosa, P. fluorescens, P. putida; y ocho al género Bacillus, representado por las especies $B$. amyloliquefaciens, $B$. simplex, $B$. subtilis, $B$. thuringiensis. De igual forma, de acuerdo con los resultados de las pruebas bioquímicas rápidas, se confirmó la presencia de Enterobacter cloacae aislados EP5G1M9 y EP6G1M14 y Stenotrophomonas maltophilia aislados EP7G2M15 y EP8 B2M28. Los aislados EP1B1M8, EP2B2M4 y EP3G2M4, que de acuerdo con su perfil bioquímico mostraron características similares a la especie Geobacillus thermoglucosidasius, se identificaron molecularmente como el género Microbacterium sp., siendo los aislados EP1B1M8 y EP2B2M4 la especie Microbacterium arborescens (Cuadro 2, Figura 1). 


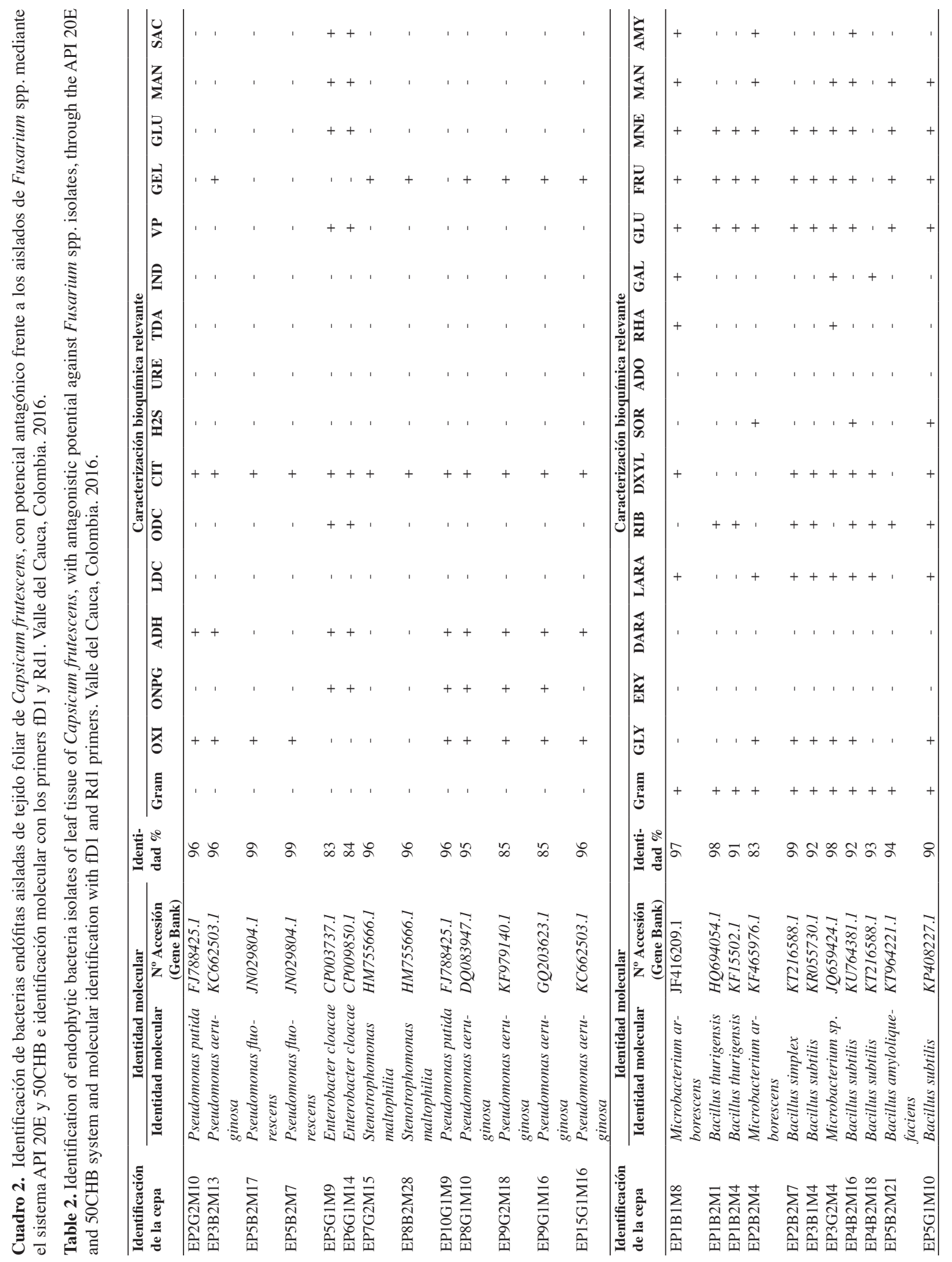




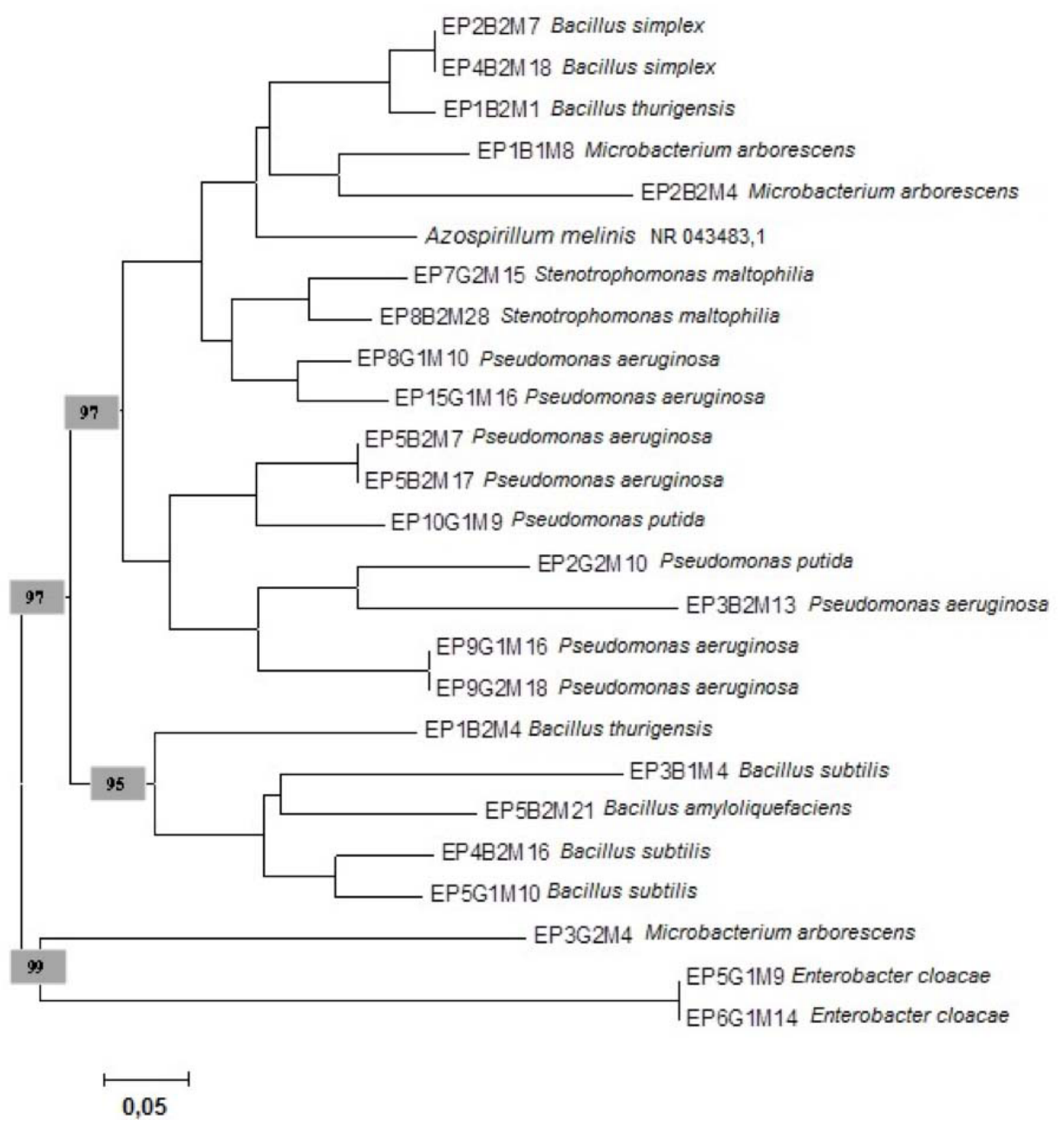

Figura 1. Árbol filogenético de las secuencias de bacterias endófitas de tejido foliar de Capsicum frutescens, con potencial antagónico frente a aislados de Fusarium spp., obtenidas mediante la amplificación del gen 16S rARN, primer universal D1, con el coeficiente de similitud del vecino más cercano y un análisis bootstrap de 1000 réplicas. Raíz de Azospirillum melinis (NR_043483,1). Valle del Cauca, Colombia. 2016.

Figure 1. Phylogenetic tree of the endophytic bacteria sequences of leaf tissue of Capsicum frutescens, with antagonistic potential against the Fusarium spp. isolates, obtained by amplification of the $16 \mathrm{~S}$ rARN gen, universal primer D1, using the similarity coefficient of the nearest neighbor and bootstrap of 1000 replicas analysis. Azospirillum melinis (NR_043483,1) root. Valle del Cauca, Colombia. 2016.

\section{Discusión}

En este estudio se obtuvieron 68 aislamientos endófitos bacterianos del tejido foliar de C. frutescens, de los cuales dieciséis presentaron porcentajes de inhibición por encima del $40 \%$. Los aislados EP5G1M10 y EP3B1M4, identificados bioquímica y molecularmente como Bacillus subtillis, demostraron inhibir a todas los aislados de Fusarium spp. a los que se expusieron, con porcentajes promedios de 76 a $86 \%$. De igual manera, los aislados EP8G1M10 y EP9G2M18, identificados bioquímica y molecularmente como P. aeruginosa, presentaron 
promedios de inhibición entre 62 y $89 \%$ frente a todos los aislados de Fusarium spp. Estos porcentajes fueron altos, comparados con estudios similares realizados en Colombia; como el de Franco-Correa (2008), Ramos y Valero (2008) y Venner y Martín (2009), quienes reportaron porcentajes de inhibición de Fusarium por bacterias rizosféricas, no mayores a $68 \%, 76 \%$ y $40 \%$, respectivamente (Venner y Martín, 2009). Estos datos coinciden con lo reportado en la literatura, en donde se describe la capacidad de estos microorganismos para controlar hongos patógenos sintetizando moléculas antifúngicas (Whipps, 2001), como quitinasas, glucanasas y proteasas que degradan y rompen las paredes celulares de los hongos (Lugtenberg y Kamilova, 2009).

Diferentes estudios con especies del género Pseudomonas han mostrado la capacidad de este microorganismo para ser empleado como antagonista. En un estudio realizado en 1991, Toyoda y Utsumi, demostraron que cepas de Pseudomonas cepacia y Pseudomonas solanacearum fueron capaces de hidrolizar el ácido fusárico, el cual es el agente causante del marchitamiento por infección de Fusarium (Toyoda y Utsumi, 1991). Además, Cordero et al. (2014) evidenciaron que Pseudomonas fluorescens MGR12, en crecimiento sobre medio mínimo y enriquecido, fue capaz de producir compuestos volátiles que inhiben el crecimiento de Fusarium proliferatum (Cordero et al., 2014). La cepa silvestre P. aeruginosa PA1201 inhibe el crecimiento de una gran variedad de patógenos, entre ellos Fusarium graminearum; debido a la producción de altos niveles de ácido phenazine-1-carboxylic (PCA), fungicida conocido como shenqinmycina, y la producción de phenazine-1-carboxaminde (PCN), un derivado de PCA (Zhou et al., 2016). Recientemente, en un estudio realizado por Uzair et al. (2018), en el que se aislaron cuatro cepas de Pseudomonas con actividad de biocontrol frente a hongos fitopatógenos como Rhizopus microsporus, Fusarium oxysporum, Aspergillus niger, Alternaria alternata, y Penicillium digitatum; se demostró que la cepa PS24, identificada como Pseudomonas aeruginosa, produjo diferentes antibióticos, dentro de los cuales se destacó la Pirrolnitrina. Este antibiótico exhibió actividades antifúngicas que resultaron positivas para el gen de cianuro de hidrógeno sintasa $H c n$ BC. Por lo que los autores argumentan el empleo de esta cepa como biocontrolador de hongos fitopatógenos (Uzair et al., 2018).

La producción de sideróforos, tiene un efecto inhibitorio en hongos fitopatógenos, debido a la competencia por captación de hierro (Kloepper et al., 1980; Loper y Schroth, 1986). Esta característica de biocontrol, se puede confirmar con los resultados de la presente investigación; en donde los aislados de Pseudomonas (EPG2M18 y EP8G1M10) con mayor porcentaje de inhibición frente a Fusarium, identificados molecularmente y bioquímicamente como $P$. aeruginosa, produjeron sideróforos. Sin embargo, los demás asilados en los que se evidenció producción de sideróforos, identificados como P. aeruginosa (EP9G1M16 y EP15G1M16) у P. putida (EP10G1M9), mostraron un efecto inhibitorio menor al $50 \%$. Estos resultados confirman que Pseudomonas puede establecer más de un mecanismo de inhibición frente a Fusarium y comprueban el gran potencial biotecnológico de cepas silvestres de este microorganismo para el biocontrol de hongos fitopatógenos.

Las bacterias del género Bacillus, se han caracterizado por tener una acción directa e indirecta como bacterias promotoras de crecimiento vegetal (Lugtenberg y Kamilova, 2009; Sarabia et al., 2010). La especie B. subtilis, en especial, presenta gran interés en el biocontrol de hongos y bacterias fitopatógenas (Fernández-Larrea, 2001). Esta bacteria produce metabolitos secundarios como lipopéptidos cíclicos entre los que se encuentran la Iturina, fengycina y surfactina, que han demostrado un gran potencial de aplicación tanto en la industria agrícola como la industria farmacéutica, debido a su dinámica como biosurfactantes (Banat et al., 2000). Además, estos compuestos contribuyen en el crecimiento y supervivencia de la bacteria, al permitirle la formación de biopelículas, el establecimiento de comunidades multicelulares, y la motilidad tipo swarming; características que favorecen el aumento de la colonización de la bacteria (Sarwar et al., 2018). Sobre Fusarium, Mahmoud (2016) comprobó la acción inhibitoria de cepas silvestres de B. subtilis, aisladas de granos de trigo, sobre el crecimiento de diferentes cepas patógenas de Fusarium graminearum productoras de la fusariosis de la espiga. La acción antagónica de $B$. subtilis sobre $F$. graminearum fue significativa, al igual que la reducción de la fusariosis de la espiga en cultivo in vitro en invernadero (Mahmoud, 2016). Asimismo, Tejera-Hernández et al. (2011) destacaron el gran potencial 
de $B$. subtilis, como inhibidor de Fusarium oxysporum, debido a la producción lipasas, proteasas y $\beta$-glucanasas y quitinasas (Tejera-Hernández et al., 2011).

La producción de sideróforos es una característica que se ha descrito mayormente en el género Pseudomonas, sin embargo, existen evidencias de la producción de sideróforos como bacilibactina, ácido itoico y ácido 2,3-dihidroxibenzoico por parte de la especie B. subtilis (Ito, 1993; Dertz et al., 2006). Los resultados del presente estudio, mostraron que los aislados con mayores porcentajes de inhibición, sobre las seis cepas de Fusarium spp, fueron identificados como B. subtilis, no obstante, en estas cepas no se evaluó la producción de sideróforos, por lo que no se descarta su producción y acción frente a Fusarium spp. Sin embargo, es necesario realizar más pruebas para comprobar cuál fue el mecanismo de inhibición que emplearon estos aislados antagonistas sobre los hongos.

Otras bacterias endófitas que presentaron porcentajes de inhibición mayores al $50 \%$, frente al menos un aislado de Fusarium spp, fueron las Gram negativas Enterobacter cloacae EP6G1M1 y Stenotrophomonas maltophilia EP8B2M28, las cuales han sido reportadas anteriormente como bacterias con potencial de biocontrol frente a Ralstonia solanacearum (Liu et al., 2012; Elhalag et al., 2015). La bacteria Gram positiva Microbacterium arborescens EP2B2M4, mostró promedios de porcentaje de inhibición mayores a $60 \%$ frente a dos aislados de Fusarium spp. Esta bacteria ha sido reportada como promotora de crecimiento vegetal por su capacidad de producir diferentes metabolitos benéficos para la planta (Malik y Sindhu, 2011; Jorquera et al., 2012; Morango-González et al., 2015) y por su capacidad para solubilizar fosfatos en especies como Capscium annuит, Lupinus montanus, entre otros (Oliveira et al., 2009; Bhattacharyya y Jha, 2012; Babana et al., 2013; Molina-Romero et al., 2015).

\section{Conclusiones}

Del tejido foliar endófito de ají tabasco ( $C$. frutescens) se identificaron cinco géneros de bacterias endófitas con capacidad antagónica frente a Fusaruim spp., correspondientes a Pseudomonas, Bacillus, Microbacterium, Enterobacter, Stenotrophomonas.

La morfología, perfil bioquímico y caracterización molecular, permitió determinar que los géneros Pseudomonas y Bacillus, fueron los más representativos en cuanto abundancia y capacidad antagónica frente a Fusarium spp. De estos, las especies Pseudomonas aeruginosa y Bacillus subtilis fueron las más predominantes en el estudio.

Las especies Enterobacter cloacae, Microbacterium arborescens, y Stenotrophomonas maltophilia, son nuevos reportes de bacterias asociadas al tejido vegetal endófito de plantas de ají tabasco $C$. frutescens, en el Valle del Cauca, Colombia.

Los resultados encontrados en este estudio indican, que los aislados de bacterias endófitas con poder antagónico sobre Fusarium, aisladas en ají tabasco (Capsicum frutescens), pueden ser una buena alternativa como biocontroladores de patógenos como Fusarium y presentar un gran potencial de empleo como PGPB para el mejoramiento de la productividad en este cultivo.

\section{Agradecimientos}

Los autores agradecen a los grupos de investigación "Protección Vegetal para el Mejoramiento de la productividad" de la Universidad Nacional de Colombia sede Palmira, "Biología de plantas y Microorganismos" de la Universidad del Valle y "Microambiente Libre" de la Universidad Libre, Seccional Cali, por su colaboración en el desarrollo de la investigación. 


\section{Literatura citada}

Acosta, T., A. Avellaneda, J. Cuervo, y L. Sánchez. 2007. Evaluación de microbiota de tomillo (Thymus vulgaris), como aporte al manejo agroecológico de aromáticas en invernaderos de la Universidad Nacional. En: Universidad Nacional de Colombia, editor, Perspectivas del agronegocio de hierbas aromáticas culinarias y medicinales. Universidad Nacional de Colombia, Bogotá, COL. p. 135-138.

Agrios, G.N. 2005. Plant pathology. $5^{\text {th }}$ ed. Elsevier Academic Press, Burlington, MA, USA.

Amaresan, N., V. Jayakumar, and N. Thajuddin. 2014. Isolation and characterization of endophytic bacteria associated with chilli (Capsicum annuum) grown in coastal agricultural ecosystem. Ind. J. Biotechnol. 13:247-255.

Andrés-Ares, J.L., A. Rivera-Martínez, F. Pomar-Barbeito, and J. Fernández-Paz. 2005. Telluric pathogens isolated from blighted pepper (Capsicum annuum L.) plants in northwestern Spain. J. Agric. Res. 3:326-330. doi:10.5424/sjar/200533-161

Babana, A.H., A. Dicko, K. Maïga, and D. Traoré. 2013. Characterization of rock phosphate-solubilizing microorganisms isolated from wheat (Triticum aestivum L.) rhizosphere in Mali. J. Microbiol. Microbial Res. 1(1):1-6.

Banat, I.M., R.S. Makkar, and S.S. Cameotra. 2000. Potential commercial applications of microbial surfactants. Appl. Microbiol. Biotechnol. 53:495-508. doi:10.1007/s002530051

Barquero, M., A. Terrón, E. Velázquez, and F. González-Andrés. 2016. Biocontrol of Fusarium oxysporum f.sp. phaseoli and Phytophthora capsici with autochthonous endophytes in common bean and pepper in Castilla y León (Spain). In: F. González-Andrés, and E. James, editors, Biological nitrogen fixation and beneficial plant-microbe interaction. Springer, Cham, SUI. p.221-235. doi: 10.1007/978-3-319-32528-6_19

Benítez, S., J. Bentley, P. Bustamante, L.C. Sánchez, y L. Corrales. 2007. Aislamiento de los microorganismos cultivables de la rizósfera de Ornithogalum umbellatum y evaluación del posible efecto biocontrolador en dos patógenos del suelo. Nova 5:147-153.

Bhattacharyya, P.N., and D.K. Jha. 2012. Plant growth promoting rhizobacteria (PGPR): emergence in agriculture. World J. Microbiol. Biotechnol. 28:1327-1350. doi:10.1007/s11274-011-0979-9

Brader, G., S. Compant, B. Mitter, F. Trognitz, and A. Sessitsch. 2014. Metabolic potential of endophytic bacteria. Curr. Opin. Biotechnol. 27:30-37. doi:10.1016/j.copbio.2013.09.012

Clavijo-Castro, S. 2014. Búsqueda de resistencia a la pudrición causada por Fusarium spp. en Capsicum. Tesis MSc., Universidad Nacional de Colombia, Palmira, COL.

Cordero, P., A. Príncipe, E. Jofré, G. Mori, and S. Fischer. 2014. Inhibition of the phytopathogenic fungus Fusarium proliferatum by volatile compounds produced by Pseudomonas. Arch. Microbiol. 196:803-809. doi:10.1007/s00203-014-1019-6

Dertz, E.A., A. Stintzi, and K.N. Raymond. 2006. Siderophore-mediated iron transport in Bacillus subtilis and Corynebacterium glutamicum. J. Biol. Inorg. Chem. 11:1087-1097. doi:10.1007/s00775-006-0151-4

Edward, E.J., W.S. King, S.L.C. Teck, M. Jiwan, Z.F.A. Aziz, F.R. Kundat, O.H. Ahmed, and N.M.A. Majid. 2013 . Antagonistic activities of endophytic bacteria against Fusarium wilt of black pepper (Piper nigrum). Int. J. Agric. Biol. 15:291-296.

Elhalag, K.M., N.A. Messiha, H.M. Emara, and S.A. Abdallad. 2015. Evaluation of antibacterial activity of Stenotrophomonas maltophilia against Ralstonia solanacearum under different application conditions. J. Appl. Microbiol. 120:1629-1645. doi:10.1111/jam.13097

Fernández-Larrea, O. 2001. Microorganismos antagonistas para el control fitosanitario. Manejo Integrado Plagas 62:96-100. 
Franco-Correa, M. 2008. Evaluación de caracteres PGPR en actinomicetos e interacciones de estas rizobacterias con hongos formadores de micorrizas. Tesis Dr., Universidad de Granada, ESP.

Glick, B.R. 2012. Plant growth-promoting bacteria: mechanisms and applications. Scientifica 2012:963401. doi:10.6064/2012/963401.

Glick, B.R. 2015. Beneficial plant-bacterial interactions. Springer International Publishing, SUI.

González, I., Y. Arias, y B. Peteira. 2012. Aspectos generales de la interacción Fusarium oxysporum f. sp. lycopersici-tomate. Rev. Protección Veg. 27:1-7.

Guigón-López, C., y P.A. González-González. 2001. Estudio regional de las enfermedades del chile (Capsicum annuum L.) y su comportamiento temporal en el sur de Chihuahua, México. Rev. Mex. Fitopatol. 19:49-56.

Ito, T. 1993. Enzymatic determination of itoic acid, a Bacillus subtilis siderophore, and 2,3-dihydroxybenzoic acid. Appl. Environ. Microbiol. 59:2343-2345.

Islek, C., and B. Turkyilmaz-Unal. 2015. Copper toxicity in Capsicum annuum: Superoxide dismutase and catalase activities, phenolic and protein amounts of in-vitro-grown plants. Polish J. Environ. Stud. 24(6):47-51 . doi:10.15244/pjoes/59035

Jorquera, M.A., B. Shaharoona, S.M. Nadeem, M. De-la-Luz-Mora, and D.E. Crowley. 2012. Plant growth-promoting rhizobacteria associated with ancient clones of creosote bush (Larrea tridentata). Microbial Ecol. 64:1008-1017. doi:10.1007/s00248-012-0071-5

Kloepper, J.W., J. Leong, M. Teintze, and M.N. Schroth. 1980. Enhanced plant growth by siderophores produced by plant growth-promoting rhizobacteria. Nature 286:885-886. doi:10.1038/286885a0

Kloepper, J.W., R. Rodríguez-Kábana, A.W. Zehnder, J.F. Murphy, E. Sikora, and C. Fernández. 1999. Plant root-bacterial interactions in biological control of soilborne diseases and potential extension to systemic and foliar diseases. Australas. Plant Pathol. 28:21-26. doi:10.1071/AP99003

Leslie, J.F., and B.A. Summerell. 2006. The Fusarium Laboratory Manual. Blackwell Publishing, Ames, IA, USA.

Liu, W.Y., K.M. Chung, C.F. Wong, J.W. Jiang, R.K. Hui, and F.C. Leung. 2012. Complete genome sequence of the endophytic Enterobacter cloacae subsp. cloacae strain ENHKU01. J. Bacteriol. 194:5965-5965. doi:10.1128/JB.01394-12

Loper, J.E., and M.N. Schroth. 1986. Importance of siderophores in microbial interactions in the rizosphere. In: T.R. Swinburne, editor, Iron, siderophores and plant diseases. Springer, Boston, MA, USA. p. 85-98

Lugtenberg, B., and F. Kamilova. 2009. Plant-growth-promoting rhizobacteria. Annu. Rev. Microbiol. 63:541-556. doi:10.1146/ annurev.micro.62.081307.162918

Mahmoud, A.F. 2016. Genetic variation and biological control of Fusarium graminearum isolated from wheat in Assiut-Egypt. Plant Pathol. J. 32:145-156. doi:10.5423/PPJ.OA.09.2015.0201

Malik, D.K., and S.S. Sindhu. 2011. Production of indole acetic acid by Pseudomonas sp.: effect of coinoculation with Mesorhizobium sp. Cicer on nodulation and plant growth of chickpea (Cicer arietinum). Physiol. Mol. Biol. Plants 17:2532. doi:10.1007/s 12298-010-0041-7

Merino, L.A., y G. Giusiano. 2011. Manual de métodos moleculares para estudios microbiológicos. Asociación Argentina de Microbiología, Buenos Aires, ARG.

Molina-Romero, D., M.R. Bustillos-Cristales, O. Rodríguez-Andrade, Y.E. Morales-García, Y. Santiago-Saenz, M. CastañedaLucio, y J. Muñoz-Rojas. 2015. Mecanismos de fitoestimulación por rizobacterias, aislamientos en América y potencial biotecnológico. Biológicas 17(2):24-34. 
Morales-Rondón, V., y M. Rodríguez-González. 2006. Hongos endófitos en plantaciones de mango `Haden’ de la planicie de Maracaibo, Venezuela. Rev. Fac. Agron. 23:273-284.

Morango-González, A.M., D. Espinoza-Victoria, y F.C. Gómez-Merino. 2015. Eficiencia de las bacterias promotoras del crecimiento vegetal (BPCV) en caña de azúcar. Rev. Terra Latinoam. 33:321-330.

Muhammad, U., F. Mudassar, F. Kanza, S. Muhammad, A. Qurban, R. Bushra, Q. Abdul A. Idrees, and H. Tayyab. 2016. Phytophthora capsici on chili pepper (Capsicum annuum L.) and its management through genetic and bio-control: a review. Zemdirbyste-Agriculture 103:419-430. doi:10.13080/z-a.2016.103.054

Narayan C., H. Seung, X. Jian, and H. Seung. 2013. Assemblages of endophytic bacteria in chili pepper (Capsicum annuum L.) and their antifungal activity against phytopathogens in vitro. POJ 6:441-448

Nongkhlaw, W., F. Mary, and S.R. Joshi. 2014. Epiphytic and endophytic bacteria that promote growth of ethnomedicinal plants in the subtropical forests of Meghalaya, India. Rev. Biol. Trop. 62:1295-1308. doi:10.15517/rbtv62i4.12138

Ochoa, L., y M. Lundy. 2002. El caso de la producción de pasta de ají para exportación del Valle del Cauca, Colombia. FAO, CHI.

Oliveira, C.A., V.M.C. Alves, I.E. Marriel, E.A. Gomes, M.R. Scotti, N.P. Carneiro, R. Guimarães, R. Shaffert, and N.M.H. Sá. 2009. Phosphate soconlubilizing microorganisms isolated from rhizosphere of maize cultivated in an oxisol of the Brazilian Cerrado Biome. Soil Biol. Biochem. 41:1782-1787. doi:10.1016/j.soilbio.2008.01.012

Peña, H.B., e I. Reyes. 2007. Aislamiento y evaluación de bacterias fijadoras de nitrógeno y disolventes de fosfatos en la promoción del crecimiento de la lechuga (Lactuca sativa L.). Interciencia 32:560-565.

Pérez, A., C. Pérez, y L. Chamorro. 2013. Diversidad de bacterias endófitas asociadas a cultivo de arroz en el departamento de Córdoba-Colombia: estudio preliminar. Rev. Colomb. Cienc. Anim. 5:83-92. doi:10.24188/recia.v5.n1.2013.473

Pérez-Hernández, A., Y. Serrano-Alonso, M.I. Aguilar-Pérez, R. Gómez-Uroz, and J. Gómez-Vázquez. 2014. Damping-off and root rot of pepper caused by Fusarium oxysporum in Almeria Province, Spain. Plant Dis. 98:1159. doi:10.1094/PDIS02-14-0212-PDN

Puri, A., K.P. Padda, and C.P. Chanway. 2018. Nitrogen-fixation by endophytic bacteria in agricultural crops: recent advances. In: S.F. Amanullah, editor, Nitrogen in agriculture. IntechOpen, London, GBR. p. 73-94. doi:10.5772/intechopen.71988

Rajkumara, M., N. Aea, and H. Freitas. 2009. Endophytic bacteria and their potential to enhance heavy metal phytoextraction. Chemosphere 77:153-160. doi:10.1016/j.chemosphere.2009.06.047

Ramos, M., y C. Valero. 2008. Aislamiento de rizobacterias promotores de germinación de uchuva (Physalis peruviana L.) y antagónica frente a Fusarium oxyporum. Pontificia Universidad Javeriana, Bogotá, COL.

Reinhold-Huker, B., and T. Hurek. 1998. Interactions of grameneous plant with Azoarcus spp., and other diazotrophic, identification, localization and perspective to study their function. Crit. Rev. Plant Sci. 17:29-54. doi:10.1080/07352689891304186

Santoyo, G., G. Moreno-Hagelsiebb, M.C. Orozco-Mosquedac, and B.R. Glickc. 2015. Plant growth-promoting bacterial endophytes. Microbiol. Res. 183:92-99. doi:10.1016/j.micres.2015.11.008

Sarabia, M., R. Madrigal, M. Martínez, e Y. Carreón. 2010. Plantas, hongos micorrízicos y bacterias: su compleja red de interacciones. Biológicas 12(1):65-71.

Smith, S.A., D.C. Tank, L.A. Boulanger, C.A. Bascom-Slack, K. Eisenman, D. Kingery, B. Babbs, K. Fenn, J.S. Greene, B.D. Hann, J. Keehner, E.G. Kelley-Swift, V. Kembaiyan, S.J. Lee, P. Li, D.Y. Light, E.H. Lin, C. Ma, E. Moore, M.A. Schorn, D. Vekhter, P.V. Nunez, G.A. Strobel, M.J. Donoghue, and S.A. Strobel. 2008. Bioactive endophytes warrant intensified exploration and conservation. PLoS ONE 3(8):e3052. doi:10.1371/journal.pone.0003052 
Sturz, A.V., and J. Nowak. 2000. An endophytic community of rhizobacteria and the strategies requires to create yield enhancing associations with crops. Appl. Soil Ecol. 15:183-190. doi:10.1016/S0929-1393(00)00094-9

Sarwar, A., G. Brader, E. Corretto, G. Aleti, M. Abaidullah, A. Sessitsch, and F.Y. Hafeez. 2018. Qualitative analysis of biosurfactants from Bacillus species exhibiting antifungal activity. PLoS ONE 13(6):e0198107. doi:10.1371/journal. pone.0198107

Tejera-Hernández, B., M. Rojas-Badía, y M. Heydrich-Pérez. 2011. Potencialidades del género Bacillus en la promoción del crecimiento vegetal y el control biológico de hongos fitopatógenos. Rev. CENIC Cienc. Biol. 42:131-138.

Toyoda, H., and R. Utsumi. 1991. US4988586A: Method for the prevention of Fusarium diseases and microorganisms used for the same. Daikin Industries Ltd., USA.

Turlier, M.F., A. Epavier, and C. Alabouvette. 1994. Early dynamic interactions between Fusarium oxysporum f.sp. lini and the roots of Linus usitatissimum as revealed by transgenic GUS-marked hyphae. Can. J. Bot. 72:1605-1612. doi:10.1139/ b94-198

Uzair, B., R. Kausar, S.A. Bano, S. Fatima, M. Badshah, U. Habiba, and F. Fasim. 2018. Isolation and molecular characterization of a model antagonistic Pseudomonas aeruginosa divulging in vitro plant growth promoting characteristics. BioMed Res. Int. 2018:6147380. doi:10.1155/2018/6147380

Velasco-Belalcazar, M. 2016. Caracterización de bacterias antagónicas a Fusarium sp., causante de la marchitez vascular de Capsicum frutescens en Guacarí y Bolivar, Valle del Cauca. Tesis MSc., Universidad Nacional de Colombia, Palmira, COL.

Venner, C., y M.J. Martín. 2009. Aislamiento y selección de rizobacterias promotoras de crecimiento vegetal en cultivos de uchuva (Physalis peruviana L.) con capacidad antagónica frente a Fusarium sp. Trabajo de grado, Pontificia Universidad Javeriana, Bogotá, COL.

Walia, A., S. Guleria, A. Chauhan, and P. Mehta. 2017. Endophytic bacteria: Role in phosphate solubilization. In: D. Maheshwari, and K. Annapurna, editors, Endophytes: Crop productivity and protection. Vol. 16. Sustainable development and biodiversity. Springer, Cham, SUI. p. 61-93. doi:10.1007/978-3-319-66544-3_4

Weisburg, W.G., S.M. Barns, D.A. Pelletier, and D.J. Lane. 1991. 16S ribosomal DNA amplification for phylogenetic study. J. Bacteriol. 173:697-703. doi:10.1128/jb.173.2.697-703.1991

Whipps, J.M. 2001. Microbial interactions and biocontrol in the rhizophere. J. Exp. Bot. 52:487-511. doi:10.1093/jexbot/52. suppl_1.487

Zhou, L., H.X. Jiang, S. Sun, D.D. Yang, K.M. Jin, W. Zhang, and Y.W. He. 2016. Biotechnological potential of a rhizosphere Pseudomonas aeruginosa strain producing phenazine-1-carboxylic acid and phenazine-1-carboxamide. World J. Microbiol. Biotechnol. 32(3):50. doi:10.1007/s11274-015-1987-y 